\title{
Design de aplicativos móveis voltados para saúde: framework com foco em persuasão
}

\author{
Designing health mobile applications: framework focusing on persuasion
}

\author{
Maria Luiza Bergo Demonte, Virginia Tiradentes Souto
}

\begin{abstract}
framework, modelo, persuasão, design e saúde
Os dispositivos móveis permitem que as pessoas estejam constantemente conectadas a vários espaços simultaneamente, facilitando o consumo e a produção de informação. A visibilidade e vigilância proporcionada por esses dispositivos torna-os propícios para o monitoramento da saúde. A partir desse panorama começaram a surgir diversos estudos na área de saúde e tecnologia para se tentar compreender e criar sistemas tecnológicos persuasivos que são efetivos. Este artigo tem como objetivo desenvolver um framework para auxiliar na concepção, análise e compreensão de aplicativos móveis relativos a saúde, com enfoque na persuasão. O Framework é dividido em três componentes principais: contexto do usuário, persuasão e design de interação. O desenvolvimento do framework é apresentado com a revisão da literatura que serviu de base para a sua construção.
\end{abstract}

framework, model, persuasion, design and health

Mobile devices allow people to be constantly connected to multiple spaces simultaneously, facilitating the consumption and production of information. The visibility and surveillance provided by these devices makes them suitable for monitoring health. From this panorama began to appear several studies in the area of health and technology to try to understand and create persuasive technology systems that are effective. This article aims to develop a framework to assist in the design, analysis and understanding of mobile applications for the practice of healthy habits, focusing on persuasion. The Framework for the Design of mobile applications related to health focusing on persuasion is divided into three main components: health habits, persuasion and interaction design. The development of the framework is presented with the literature review that was the basis for its construction.

\section{Introdução}

Os dispositivos móveis permitem que as pessoas estejam constantemente conectadas a vários espaços simultaneamente, facilitando o consumo e a produção de informação. A visibilidade e vigilância proporcionada por esses dispositivos torna-os propícios para o monitoramento da saúde. A utilização dos dispositivos móveis nesse contexto pode ser explicada pela convergência de três fatores: o aumento das doenças crônicas, diminuição do acesso aos cuidados clínicos, e as inovações no âmbito das tecnologias móveis (Morris, 2012). A partir desse panorama começaram a surgir diversos estudos na área de saúde e tecnologia para se tentar compreender e criar sistemas tecnológicos persuasivos que são efetivos.

Quando se fala em aplicativos móveis persuasivos que promovem hábitos saudáveis destacam-se três temas de grande importância: a persuasão, a saúde e o design de interação. O papel da persuasão nesse contexto é servir como forma de incentivo à prática de hábitos 
saudáveis. As tecnologias persuasivas são sistemas computacionais interativos intencionalmente criados para mudar o comportamento ou atitude de uma pessoa. Elas são onipresentes, vive-se envolto em produtos digitais concebidos para mudar pensamentos e comportamentos (Fogg, 1998). O design de interação tem grande influência no trabalho de persuasão, afinal, não existe persuasão sem interação, Compreender o usuário, suas necessidades e a sua experiência com o produto é imprescindível para que a persuasão seja satisfatória.

Para desenvolver produtos interativos é necessário considerar quem irá utilizá-los, como irá utilizá-los, onde irá utilizá-los e compreender o tipo de atividade exercida enquanto o produto está em uso. A adequação de diferentes tipos de interface irá depender das atividades apoiadas (Rogers, Sharp e Preece, 2011). Para que a tecnologia seja considerada persuasiva ela deve ser projetada com o propósito de mudar uma atitude ou comportamento de uma forma pré-determinada, nesse caso incentivar hábitos saudáveis (Fogg, 1999).

Com o objetivo de analisar e compreender cada um dos temas que envolvem a persuasão, a saúde e o design de interação pesquisadores desenvolveram modelos e frameworks. Estes foram criados a partir de teorias existentes em diversas áreas do conhecimento tais como da psicologia, da comunicação, do design entre outras áreas correlatas. Apesar da existência de diversos frameworks e modelos que podem ser utilizados para analisar e compreender os fatores relacionados a aplicativos voltados a prática de hábitos saudáveis, em sua maioria estão relacionados a aspectos da área da psicologia e da tecnologia. Aparentemente poucos estudos foram feitos com ênfase no design de interação.

Este artigo tem como objetivo desenvolver um framework para auxiliar na concepção, análise e compreensão de aplicativos móveis relativos à prática de hábitos saudáveis, com enfoque na persuasão. $O$ framework foi desenvolvido a partir de uma revisão de literatura de frameworks relacionados.

\section{Frameworks relacionados a aplicativos móveis voltados para saúde}

O propósito de modelos e frameworks é descrever, explicar ou prever. São ferramentas para estruturar o pensamento e ação sobre um problema. Eles fornecem uma base racional, para justificar decisões e explicar resultados (llott et al., 2013).

Frameworks podem ser definidos como um conjunto de conceitos inter-relacionados e/ou de questões específicas que se destina a informar um domínio ou área em particular (Preece, Rogers. Sharp, 2011). Normalmente, são derivados de uma teoria ou de um conjunto de hipóteses (Rogers e Muller, 2006). Eles servem como base para a identificação e compreensão de padrões ou correlações de interconexões entre eventos, ideias, observações, conceitos, conhecimentos, interpretações, etc. (Svinick, 2010). Ainda, os frameworks são utilizados com funções diferentes como, por exemplo: modelos preditivos, explicativos, guias instrucionais e orientações; e podem funcionar de formas variadas: incluindo etapas, questões, conceitos, desafios, princípios, heurísticas e problemas (Rogers e Muller, 2006).

Já modelos são construções abstratas, conceituais que representam processos, variáveis e relações, sem fornecer orientações especificas ou práticas para a implementação (Tomhave, 2005). Frameworks e modelos podem ser utilizados isoladamente ou combinados seja em forma de guia, de implementação de serviços, de estudos de investigação entre outros (Ilott, Gerrish, Laker, Bray, 2013).

Existem diversos frameworks na literatura relacionados a aspectos cognitivos, persuasivos e tecnológicos de produtos digitais. Frameworks e modelos relacionados e/ou voltados a aplicativos móveis com enfoque na prática de hábitos saudáveis foram selecionados para este estudo. Os frameworks/modelos foram divididos em três categorias: saúde, persuasão e design de interação. Para cada categoria são descritos dois frameworks e/ou modelos que mais se aproximavam do tema da pesquisa. Importante realçar que estes não foram os únicos frameworks e modelos utilizados na construção do framework proposto neste estudo, mas sim os mais relevantes. 


\subsection{Saúde}

Estão descritos abaixo um modelo e um conjunto de diretrizes relativos a adoção de hábitos saudáveis: o Health Befief model (HBM) originalmente proposto por Hochbaum, Rosenstock e Kegels (1950) e posteriormente revisado por Glanz, Rimer e Viswanath (2008) e e as Sete Diretrizes de Morris (2012). Assim como outros modelos sobre comportamento e saúde, como por exemplo, o 'Self-Determination Health Behaviour Model' (Ryan, 2008), o HBM apresenta percepções dos indivíduos que podem levar a adoção de hábitos saudáveis, sendo a motivação para saúde o principal foco. Com uma outra abordagem, as "Sete Diretrizes" tem como objetivo incentivar as pessoas a adquirir hábitos mais saudáveis através das tecnologias móveis. Ambas apresentam características relevantes sobre o comportamento das pessoas a partir de diferentes perspectivas em relação à prática de hábitos saudáveis e, portanto são muito relevantes no design de aplicativos móveis para a saúde.

\section{Health Befief model (BHM)}

O Health Befief model (BHM) é um modelo antigo, originalmente criado na década de 1950 (Hochbaum, Rosenstock e Kegels, 1950), foi incrementado ao longo dos anos e ainda é muito utilizado na compreensão da adoção de comportamentos saudáveis. Sua principal premissa está baseada na ideia de que o comportamento saudável é determinado por crenças pessoais ou percepções sobre determinada doença e as estratégias existentes para diminuir sua ocorrência. O modelo descrito aqui tem como base a publicação de Glanz, Rimer e Viswanath (2008).

O HBM é constituído de seis percepções pessoais dos indivíduos, são elas: (1) a seriedade percebida, essa está relacionada a percepção que o indivíduo tem da severidade da doença; (2) a suscetibilidade percebida é a percepção que o individuo possui em relação as suas chances de contrair uma doença; (3) os benefícios percebidos estão ligados as vantagens que o individuo vê em prevenir uma doença; (4) as barreiras percebidas são todos os impedimentos percebidos pelo indivíduo na ação de prevenção da doença; (5) a deixa para a ação é aquilo que irá impulsionar a mudança de comportamento; (6) a auto eficácia está relacionada a crença que o indivíduo tem de que irá conseguir realizar determinada ação para tornar sua vida mais saudável; e (7) as variáveis modificadoras são fatores pessoais de um indivíduo que afetam a adoção de um novo comportamento. Cada uma delas, seja individualmente ou combinadas, podem explicar a adoção de hábitos saudáveis por parte do individuo. Essas seis percepções podem ser modificadas por variáveis como cultura, escolaridade, experiências passadas, entre outras. Figura 1 mostra a versão original do modelo.

Figura 1. Health Belief model adaptado de Glanz, Rimer e Viswanath, 2008 (Tradução livre).

Fatores Modificadores

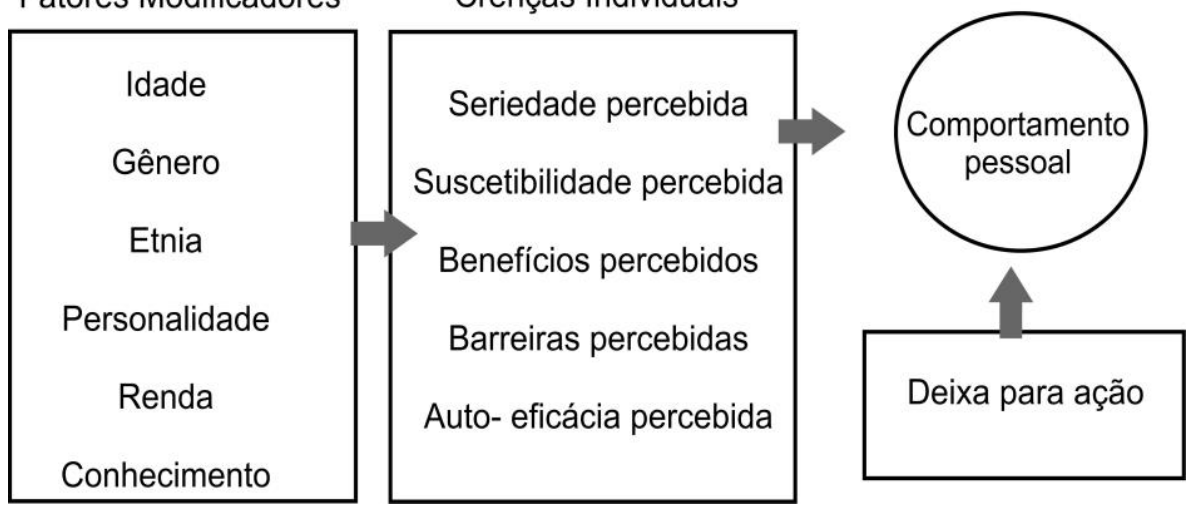


Como dito anteriormente essas percepções podem agir tanto individualmente como juntas, complementando uma a outra, anulando uma a outra ou reforçando. Esse modelo está muito ligado a mudança de hábitos das pessoas.

\section{Sete Diretrizes}

As seven guidelines (sete diretrizes) propostas por Margaret Morris (2012) assim como o modelo anterior é relativa a adoção de hábitos saudáveis porém de uma outra perspectiva. Ao invés de destacar o que leva uma pessoa a adquirir hábitos mais saudáveis, seu foco é em como incentivar essa mudança através das tecnologias móveis. As sete diretrizes são: (1) fazer as pessoas lembrarem quem elas querem ser; (2) promover uma aliança, (3) aplicar influência social, (4) mostrar às pessoas aquilo que elas podem perder, (5) colocar a mensagem onde a ação está, (6) aumentar a consciência emocional, (7) reformular desafios.

Sobre a primeira diretriz Morris (2012) explica que existem duas maneiras eficazes de recorrer aos ideais pessoais: através do contraste entre comportamento e valores como é apresentado na teoria da dissonância cognitiva ${ }^{1}$; e através de reforços positivos quando as pessoas se sentem especiais naquilo que fazem se sentem dispostas a praticar hábitos saudáveis de forma consistente. Para explicar a segunda diretriz, Morris compara a interação dos usuários e seus dispositivos com a relação entre terapeutas e seus pacientes. Os dispositivos, assim como os terapeutas, devem conhecer os objetivos de seu usuário e apontar a direção correta. Na terceira diretriz, a autora argumenta que é relevante comparar as atividades do usuário com pesquisas sobre o assunto. A quarta diretriz, refere-se as possíveis privações relacionadas a falta da saúde. Na quinta diretriz, chama atenção para a necessidade da mensagem estar coloca no contexto de uso. Na sexta diretriz, a autora chama atenção a importância da consciência emocional do usuário e a interferência na motivação do mesmo. E por último, a autora propõe que os desafios sejam reformulados, através da análise da reação das pessoas aos eventos diários.

A relevância destas diretrizes no desenvolvimento de aplicativos móveis com o foco em saúde deve-se ao fato de que elas podem auxiliar no entendimento das maneiras pelas quais os dispositivos móveis podem interagir com o usuário para motivá-lo a mudar determinada comportamento relacionado à saúde.

\subsection{Persuasão}

Existem vários frameworks e modelos voltados para persuasão com diferentes perspectivas. Alguns relativos à mudança de atitude como o Likehood model (Petty e Cacioppo, 1970) ou como os modelos apresentados abaixo. O Persuasive System Design Framework (OinasKukkonen e Harjumaa, 2009) engloba tanto a mudança de atitudes como a mudança de comportamentos já o Fogg behavior model (Fogg, 2009) que é voltado especificamente para mudança de comportamento.

Framework de Design de sistemas persuasivos (PSD)

O Persuasive Systems Design model (PSD) é um framework que aborda o processo de formulação e avaliação de sistemas persuasivos. De acordo com este modelo, a elaboração de sistemas persuasivos consiste em três passos: compreender a questão central que o contorna, o contexto que o envolve e os seus atributos (Oinas-Kukkonen e Harjumaa, 2009). Figura 2 mostra os três passos do modelo. 
Figura 2. Passos do Persuasive Systems Development (PSD). Adaptado de Oinas-Kukkonen and Harjumaa (2009). Tradução livre.

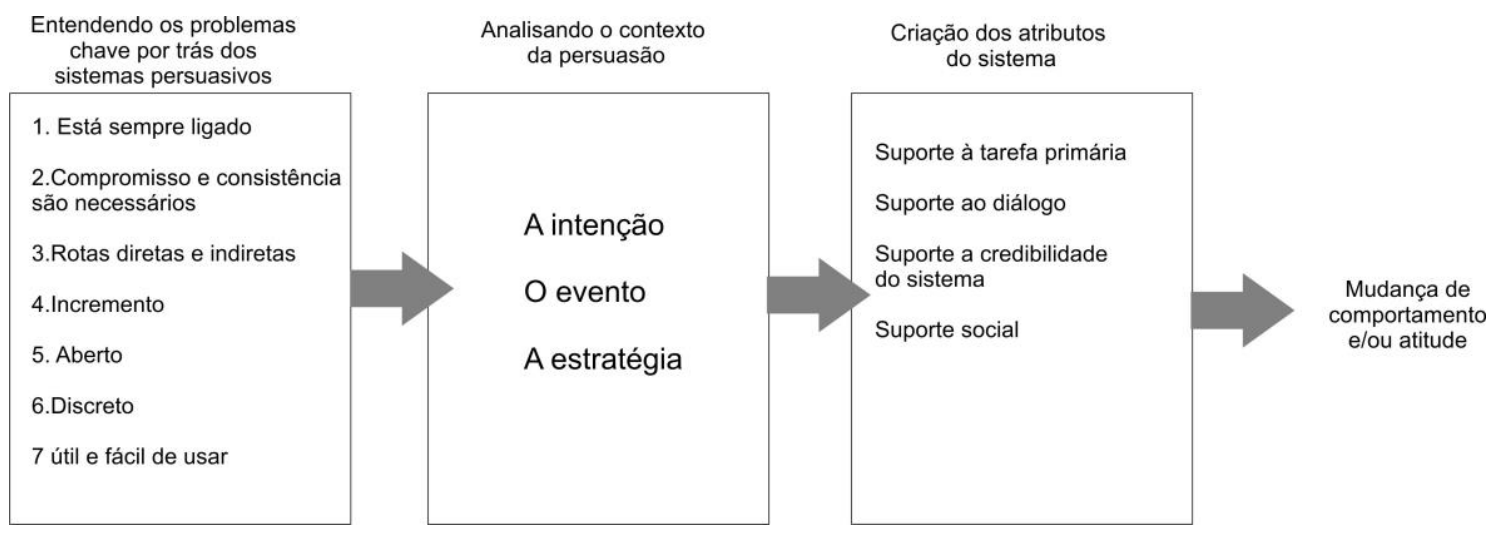

Para compreender a questão central do sistema persuasivo os autores propõem sete postulados. Os dois primeiros estão relacionados aos usuários: (1) tecnologia da informação nunca é neutra, as pessoas estão constantemente sobre a influência de algo ou alguém; (2) as pessoas gostam de ter uma perspectiva organizada e consistente sobre o mundo; caso o sistema se fundamente em um dever ou um compromisso, as pessoas estarão mais dispostas a realizá-lo.

Os outros dois postulados estão associados às estratégias persuasivas: (3) a chave para as estratégias de persuasão são as rotas diretas (i.e. pessoa avalia cuidadosamente 0 conteúdo da mensagem) e indiretas (i.e. o indivíduo é menos pensativo e utiliza pistas simples ou estereótipos para avaliar as informações); (4) a persuasão é frequentemente potencializadora, ou seja, ela gradualmente incrementa determinado comportamento.

Os últimos três postulados estão ligados aos recursos do sistema: (5) a intenção dos sistemas persuasivos deve ser clara, é importante que o usuário consiga identificar o viés do designer dentro do sistema; (6) sistemas persuasivos devem ser discretos, evitando incomodar seus usuários; (7) sistemas persuasivos devem ser úteis e fáceis de usar.

Outra etapa de grande importância é a análise do contexto da persuasão. São destacadas três maneiras de analisar esse contexto: a intenção, relativa ao objetivo do persuasor o evento, que incluí o contexto de uso do usuário e da tecnologia e a estratégia, correspondente a rota da mensagem. Por último, o modelo sugere a análise dos atributos do sistema onde são propostos 28 princípios de design para o conteúdo de sistemas persuasivos. Esses atributos são categorizados em: suporte à tarefa primária, suporte ao diálogo, suporte à credibilidade do sistema e suporte social.

PSD model é um framework que envolve passos para a criação de sistemas persuasivos em geral. Por ser amplo está voltado para a implementação de software e não para construção de interfaces. O foco do framework é a mudança de uma atitude e ou comportamento diferente do framework apresentado a seguir.

\section{Fogg behavior Model (FBM)}

Diferente do PSD model, que faz uma abordagem ampla da concepção de sistemas persuasivos, o Fogg behavior model (FBM) tem como foco o usuário. Porém, não trata do processamento de informação e nem da mudança de atitude, focando exclusivamente na mudança de comportamento ou hábito. Seu objetivo é auxiliar profissionais de diversas áreas a compreender a mudança comportamental das pessoas. Figura 3 mostra os fatores do modelo PSD e seus subcomponentes. 


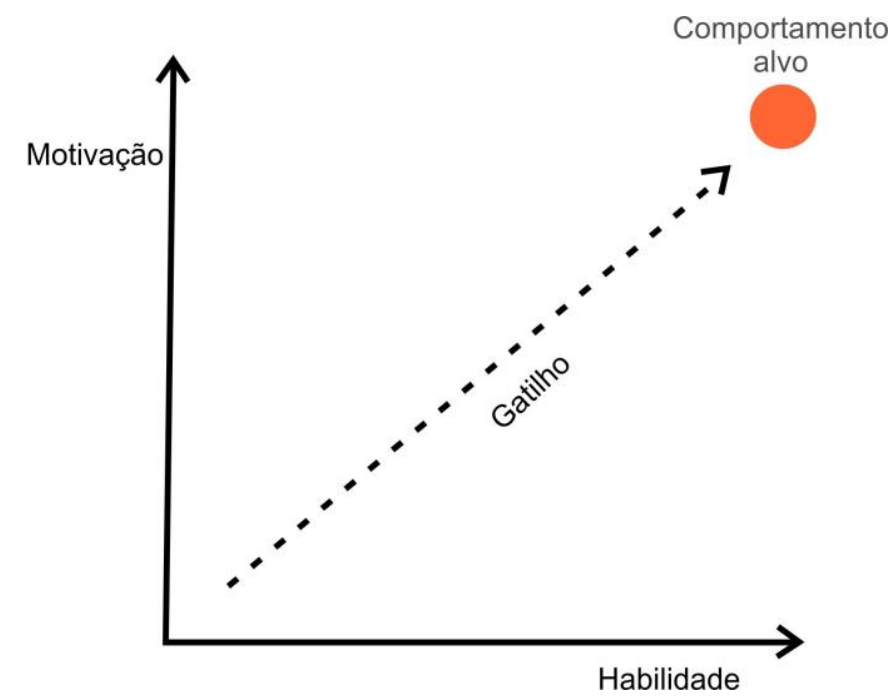

Nesse modelo a persuasão tecnológica é apresentada como aprendizagem da automação de um determinado comportamento (Fogg, 2009). O que proporciona sua mudança é um produto da convergência de três fatores: motivação, capacidade e gatilhos. Cada um deles possui subcomponentes, especificamente, três fatores motivadores centrais (motivação), seis fatores de simplicidade (capacidade), e três tipos de gatilhos (Fogg, 2011).

A motivação e a capacidade são complementares, porém não são dependentes. Em uma situação em que se tem alta motivação e alta capacidade as chances do comportamento alvo ser atingido são muito maiores (Fogg, 2002). O terceiro elemento apresentado no FBM são os gatilhos cujo papel é dar o impulso para que o comportamento seja realizado naquele determinado momento. Diferente da relação complementar apresentada pela motivação e pela capacidade, o gatilho é imprescindível para que o comportamento alvo ocorra (Fogg, 2002). Seu papel é indicar que aquele é o momento ideal para realizar determinada ação, por isso o é um elemento tão importante nesse contexto. De acordo com Fogg, existem três tipos de gatilho: o gatilho como faísca (usado quando há falta de motivação), o gatilho como facilitador (ideal para situações de alta motivação e falta de capacidade), e o gatilho como sinal (serve como um lembrete em situações de alta motivação e alta capacidade).

Fogg desenvolveu diversos estudos no campo das tecnologias persuasivas. O FBM é um framework simples de fácil compreensão, que faz parte de um sistema maior envolvendo outros frameworks com objetivo de compreender mudanças comportamentais (Fogg, 2015).

\subsection{Design de interação}

Embora frameworks relacionados ao design de interação, mais especificamente ao design dos elementos interface centrados no usuário tenham sido propostos por alguns autores (e.g. Dyson et al., 1995; Garrett, 2002) esta ainda parece ser uma área pouco investigada. Dois modelos estão descritos abaixo: o framework para o design de ambientes educacionais interativos digitais para jovens (Souto, 2014) e o User-centered design (UCD) framework for conservative environments (Tariq et al.,2009). O primeiro apesar de focado em ambientes educacionais destaca o design de interação com foco na interface, seus elementos visuais e usuários. Já o segundo é focado no processo de design de aplicações de dispositivos móveis voltadas para o cuidado com a saúde de ambientes conservadores.

Framework para o design de ambientes educacionais interativos digitais para jovens

Este framework, como o nome sugere, tem o objetivo de auxiliar na concepção de ambientes de aprendizagem interativos voltados especificamente para os jovens (Souto, 2014). Além disso, também apresenta uma estrutura voltada para a compreensão das características da 
interface e da interação dos usuários com estes ambientes. Ele é dividido em três componentes; aprendizagem, interação do usuário e visual.

Figura 4: Framework para design de ambientes educacionais interativos digitais para jovens. Adaptado de Souto (2014). Tradução livre

\begin{tabular}{|l|l|l|}
\hline Aprendizagem & Interação do usuário & Visual \\
\hline Conteúdo & Acessibilidade & Tipografia \\
Características do usuário & Interação & Ícones \\
Habilidades & Requerimentos da plataforma & $\begin{array}{l}\text { Imagens } \\
\text { Visualização da informação } \\
\end{array}$ \\
& & Estrutura da interface \\
\hline
\end{tabular}

Cada um dos desses componentes apresentados na figura 4 possuem seus subcomponentes. O primeiro componente é a aprendizagem da interface envolve os seguintes subcomponentes: (1) as características do usuário como o nome diz é relativa a informações a respeito do usuário e o meio em que vive, (2) as habilidades estão ligadas a capacidade do usuário de realizar tarefas e por fim o (3) o conteúdo que envolve o detalhes da apresentação da informação e seu formato. O segundo componente é a interação do usuário, refere-se à plataforma tecnológica utilizada e a e sua interação com o usuário. Seus subcomponentes envolvem: (1) acessibilidade, (2) usabilidade e (3) interação. E o terceiro e ultimo componente são os elementos visuais da interface. Seus subcomponentes são: (1) elementos gráficos relativos a aspectos tipográficos, imagens, ícones entre outros, (2) a estrutura de interface, que inclui aspectos como a existência de uma rede entre diferentes páginas, organização e quantidade de elementos, impressão causada pela interface, estrutura de leiaute e componentes visuais. (3) a "visualização da informação" que inclui diferentes tipos de representação visual de informações.

Apesar desse framework ser voltado para a aprendizagem de jovens muitos dos aspectos de analise de interface, usuário e características visuais são de grande relevância para outros ambientes digitais com diferentes propósitos. É importante enfatizar que mídias e públicos distintos irão exigir diferentes adaptações desses aspectos.

\section{Framework com design centrado no usuário (DCU) para ambientes conservadores}

Este framework para aplicações de e-health móvel com design centrado no usuário é especialmente focado em ambientes conservadores. O objetivo é formular as habilidades cognitivas dos usuários de maneira interativa na concepção de aplicativos de e-health assegurando uma usabilidade satisfatória do sistema. O framework é composto de quatro etapas: (1) análise de cenário, (2) conceituação do design, (3) implementação do protótipo interativo, e (4) avaliação da usabilidade.

Figura 5: Como etapas se relacionam. Adaptado de Tariq, Tanwani e Farooq (2009). Tradução livre.

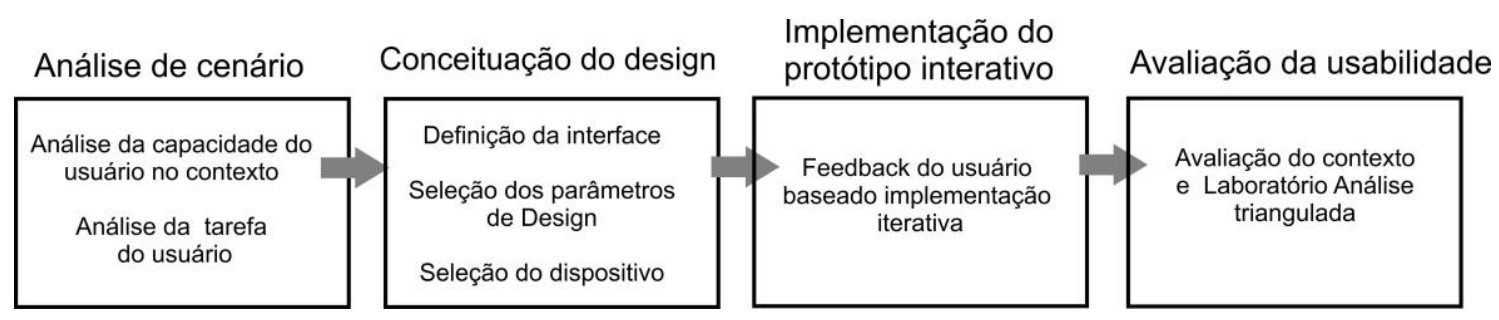

A etapa análise de cenário tem por objetivo conhecer o contexto do problema a partir da análise das capacidades do usuário. A segunda etapa, conceituação do design, mapeia as habilidades e exigências cognitivas dos usuários antes da implementação inicial. A terceira etapa, implementação do protótipo interativo, está relacionada ao desenvolvimento e implementação do aplicativo de acordo com os princípios de design. A última etapa, avaliação da usabilidade, está relacionada a uma analise mais profunda da usabilidade do aplicativo a partir de uma triangulação metodológica. 


\section{Framework para o Design de aplicativos móveis relativos à saúde com foco em persuasão}

Os frameworks e modelos descritos acima apresentaram diferentes abordagens e aspectos sobre elementos relacionados a aplicativos móveis com foco na prática de hábitos saudáveis que apesar de distintos, possuem alguns aspectos convergentes. Em todos, é possível notar um forte caráter psicológico, pois estão voltados ao engajamento do usuário. A partir dessa revisão foi possível observar diversos aspectos, conceitos e características do que pode vir a ser um aplicativo persuasivo relativo a prática de hábitos saudáveis. Como já mencionado, além dos frameworks e modelos descritos acima outros frameworks (e.g. johnson, johnson e Zhang, 2005; Ayob, Hussin e Dahlan, 2009) também foram considerados na construção do framework proposto.

O objetivo do framework aqui proposto é orientar na concepção de aplicativos mais persuasivos relacionados à prática hábitos de saudáveis. Ele é dividido em três componentes principais como mostra a figura abaixo. A seguir são explicados os componentes do framework.

Figura 6: Componentes principais do Framework para Design de aplicativos móveis de saúde com foco em persuasão.

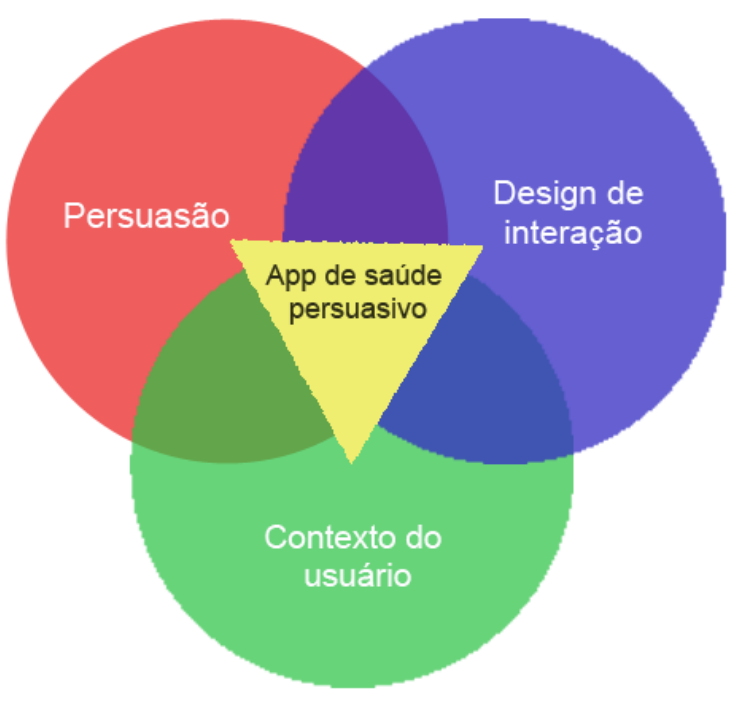

\section{1. $O$ contexto do usuário}

Como o nome sugere o contexto do usuário busca conhecer o usuário e o meio em que se encontra. Quando se trata de saúde e mudança de hábito esse aspecto é indispensável. Uma das questões mais importantes na concepção de aplicações utilizáveis é aprender sobre as pessoas que irão utiliza-las (Johnson et al. 2005). Para promover uma mudança comportamental é preciso ter conhecimentos prévios sobre o usuário, pois esses servirão como base no estabelecimento da interação entre usuário e tecnologia. De acordo com o framework HBM (Hochbaum, Rosenstock e Kegels, 1952) a percepção da pessoa pode ser influenciada por suas características individuais e por variáveis como, cultura, gênero, escolaridade, experiências passadas, habilidades, motivações entre outras. Pessoas diferentes podem ser afetadas de maneiras diferentes por fatores iguais. Ou uma mesma pessoa pode reagir de maneiras diferentes a um mesmo estimulo dependendo do estado em que se encontra.

A partir disso foram destacados alguns subcomponentes que devem ser levados em consideração. São eles: as características gerais, as necessidades, as metas, as habilidades, a motivação, e o cenário.

As características gerais tratam das informações básicas do usuário como gênero, faixa etária, escolaridade, peso, altura, patologia e suas limitações. As necessidades estão relacionadas ao estado de saúde do usuário. Diferentes condições de saúde possuem 
necessidades distintas. As metas, diferente das necessidades, estão ligadas ao objetivo do usuário. Aquilo que o usuário quer, não é necessariamente o que ele precisa. As habilidades são relativas as capacidades dos usuários alfabetização, inteligência coletiva, habilidades técnicas, habilidades de análise, habilidades para realizar tarefas. A motivação é relativa a tudo aquilo que instiga o usuário a adotar determinado comportamento naquele determinado momento. O cenário está relacionado ao contexto da rotina do usuário, dos aspectos externos que o afetam como cultura, classe social, dia-a-dia.

\subsection{Design de interação}

De acordo com Fogg (1999), para que uma tecnologia seja considerada persuasiva ela deve ser intencionalmente desenvolvida para mudar as atitudes e ou comportamentos de uma pessoa. Portanto, o Design de interação é imprescindível na persuasão móvel. Reforçando este argumento, Saffer (2010), diz que o foco do design de interação é identificar como os produtos se comportam e fornecem feedback com base no que os usuários estão fazendo. Esse componente é voltado para experiência do usuário e design de interface envolvendo um conjunto de estratégias a serem utilizadas para que o usuário tenha uma navegação satisfatória e recompensadora. Para se ter uma melhor compreensão desse processo de interação foram destacados alguns aspectos. São eles: usabilidade, interação, visualização de dados, estética e conteúdo.

A usabilidade consiste na avaliação da eficiência e eficácia do sistema a partir da análise de tarefas, suas funções, formatos de entrada e saída, restrições do sistema, categorias e do fluxo de informação. Essencialmente, a usabilidade examina a efetividade das funções e a satisfação dos usuários ao realizá-las. A interação está ligada a maneira como a interface se relaciona com o usuário e que ferramentas vão proporcionar os momentos de interação. A visualização de dados é a maneira como o usuário irá visualizar as informações, gráficos, tabelas, texto corrido, vídeo explicativo, entre outros. A estética abrange os aspectos visuais da interface como ícones, apresentação, organização dos elementos, tipo, imagem. O conteúdo está relacionado à informação que será entregue ao usuário, o tipo de informação, em que quantidade e em que momento durante a navegação. Nota-se que muitos dos subcomponentes escolhidos estão presentes no framework de Souto (2014) afinal ambos possuem um foco no design de interface e interação.

\subsection{Persuasão}

A maioria dos comportamentos relacionados à prática de hábitos saudáveis, como atividades físicas, consumo de medicamentos ou cessar um hábito ruim, não são intrinsecamente motivados ou inerentemente agradáveis. Para que a adoção de tais comportamentos seja bem sucedida, fora do ambiente de tratamento, as pessoas devem valorizar os comportamentos e endossar sua importância (Ryan, 2008). Por esse motivo, apesar da persuasão estar intimamente ligada ao design de interação, esse framework a destaca como o terceiro componente. Desta forma, ressalta a importância de aspectos persuasivos para incentivar a mudança de um comportamento ou atitude do usuário, tornando-o mais fácil e mais agradável. Porém é importante lembrar que persuasão é diferente de coerção, seu objetivo não é forçar um comportamento e sim promover uma mudança voluntaria por parte do usuário (Fogg, 2002).

Os aspectos necessários para a análise da persuasão são: a intenção, a motivação, a simplicidade, os gatilhos, o timing, a credibilidade, a influência. A intenção está relacionada ao objetivo do aplicativo em relação a sua função: mudança de atitude, comportamento ou ambos. . Enquanto a motivação do contexto do usuário é relativa aos estímulos pessoais do usuário a motivação aqui está ligada às ferramentas do aplicativo que instigam a motivação pessoal do usuário. A Simplicidade está relacionada às funções, exigências do aplicativo para realizar determinadas tarefas. Os gatilhos são propulsores para ação. O timing está ligado ao momento oportuno para ação, relativo aos gatilhos. A credibilidade está relacionada segurança que o sistema transmite para o usuário durante o uso do aplicativo. A Influência social objetiva facilitar a integração através do apoio aos usuários agindo como um ator social. 
É importante destacar que os aspectos persuasivos propostos aqui têm como objetivo analisar o nível de persuasão das interações. Por isso na prática a persuasão é paralela ao design da interação com base no contexto do usuário.

\subsection{Framework com seus componentes e subcomponentes}

Conforme demonstrado acima, a proposta do framework para o Design de aplicativos móveis relativos a prática de hábitos saudáveis contém três componentes principais e dezoito subcomponentes. A Figura 7 mostra o framework proposto com os seus componentes e seus subcomponentes.

Figura 7: Framework para Design de aplicativos móveis de saúde com foco em persuasão

\begin{tabular}{|l|l|l|}
\hline Contexto do usuário & Design de interação & Persuasão \\
\hline Características gerais & Usabilidade & Intenção \\
Necessidades & *Interação & *Motivação \\
Metas & Visualização de dados & Simplicidade \\
Habilidades & Estética & Gatilhos \\
*Motivação & Conteúdo & Timming \\
Cenário & & Credibilidade \\
& & Influências sociais \\
\hline
\end{tabular}

Este framework pode ser utilizado para analisar, comparar e investigar a utilização da persuasão em dispositivos móveis com o objetivo de incentivar a adoção de hábitos e ou atitudes mais saudáveis. Os componentes apresentados no framework tem forte relação entre si, alguns possuem relações complementares, outros de dependência. A partir de uma situação proposta são descritas e exemplificadas algumas dessas relações mostrando também uma das maneiras que o framework pode ser utilizado.

Por exemplo, em um aplicativo móvel persuasivo que incentiva a prática de corrida, o usuário receberá um relatório de desempenho cada vez que ele pratica o exercício. A partir da análise dos aspectos apresentados no framework, irão surgir questões no desenvolvimento desse relatório de desempenho.

Inicialmente é importante conhecer o usuário. Serão recolhidas suas características básicas como peso, idade, gênero, para que os dados possam ser gerados com base nessas informações. Suas habilidades de análise, alfabetização, habilidades técnicas devem ser levadas em consideração para determinar não só o conteúdo que deve ser apresentado no relatório e, mas também amaneira esses dados escolhidos serão visualizados (gráficos, tabelas, texto corrido entre outros).

Entretanto, é importante para o desenvolvedor levar em consideração os aspectos persuasivos dessa interação, se passa credibilidade, se é baseado nas motivações do usuário, se é de simples compreensão (de acordo com as habilidades do usuário) cada um desses fatores pode ser profundamente analisado. A partir desse exemplo nota-se como o modelo pode ser útil para a criação de aplicativos para dispositivos móveis relativos à prática de hábitos saudáveis também pode servir para análise de aplicativos e suas ferramentas.

\section{Considerações finais}

A partir da revisão de literatura e da análise de possibilidades, considera-se que o framework proposto neste estudo pode ser útil para analisar e compreender aplicações de dispositivos móveis relativos à saúde com caráter persuasivo. Apesar da existência de modelos e frameworks em cada um dos campos, não foi encontrado nenhum que destacasse esses três fatores simultaneamente: persuasão, prática de hábitos saudáveis e design de interação. Esse é um campo relativamente novo, extenso, e que muda com muita rapidez. Por esse motivo o framework pode ser de grande utilidade para o estudo dessa área de dispositivos móveis persuasivos relativos a prática de hábitos saudáveis que está cada vez mais presente e sempre em mutação. Servindo como um guia para designers na concepção e avaliação desses aplicativos. 
Em trabalhos futuros o framework deverá ser testado em aplicativos móveis voltados a prática de hábitos saudáveis com o objetivo de verificar a adequação dos componentes e subcomponentes, assim como a sua eficácia.

\section{Agradecimento}

Este trabalho é apoiado pela CAPES na Universidade de Brasília. Financiado pela CAPES Agência Federal de Apoio e Avaliação de Nível Superior do Ministério da Educação do Brasil.

This work is supported by CAPES at the University of Brasilia. Financed by CAPES - Brazilian Federal Agency for Support and Evaluation of Graduate Education within the Ministry of Education of Brazil.

\section{Referências}

AYOB, N.; HUSSIN, Ab Razak Che; DAHLAN, Halina Mohamed. 2009. Three layers design guideline for mobile application. In: Information Management and Engineering, 2009. ICIME'09. International Conference on. IEEE, p. 427-431.

Dyson, M. C., Andrews, M., \& Leontopoulou, S. 1995. Multimedia in Museums The Need for a Descriptive Framework. Convergence: The International Journal of Research into New Media Technologies, 1(2), 105-124.

FOGG, Brian J. 2002. Persuasive technology: using computers to change what we think and do. Ubiquity, v., n. December, p. 5.

FOGG, Brian. J.2009 . A behavior model for persuasive design. In: Proceedings of the 4th international Conference on Persuasive Technology. ACM.

FOGG, Brian J. BJ Fogg's Behavior Model, 2011. Disponível em: < http://www.behaviormodel.org/>. Acesso em 2 abr. 2015.

GARRETT, J. J. 2002. The elements of user experience: User-centered design for the web. Berkeley, CA: Peachpit Press.

GLANZ, Karen; RIMER, Barbara K.; VISWANATH, Kasisomayajula (Ed.). 2008. Health behavior and health education: theory, research, and practice. John Wiley \& Sons.

ILOTT, I., GERRISH, K., LAKER, S., \& BRAY, K. 2013. Naming and framing the problem: using theories, models and conceptual frameworks.

JOHNSON, Constance M.; JOHNSON, Todd R.; ZHANG, Jiajie. 2005. A user-centered framework for redesigning health care interfaces. Journal of biomedical informatics, v. 38, n. 1, p. $75-87$.

MORRIS, Margaret E. 2012. Motivating change with mobile: seven guidelines.interactions, $v$. 19, n. 3, p. 26-31.

OINAS-KUKKONEN, Harri; HARJUMAA, Marja.2009. Persuasive systems design: Key issues, process model, and system features. Communications of the Association for Information Systems, v. 24, n. 1, p. 28.

ROGERS, Yvonne, e MULLER, Henk. 2006. A framework for designing sensor-based interactions to promote exploration and reflection in play. International Journal of Human-Computer Studies 64.1:1-14.

ROGERS, Yvonne; SHARP, Helen; PREECE, Jenny. 2011. Interaction design: beyond humancomputer interaction. John Wiley \& Son.

Ryan, R. M., Patrick, H., Deci, E. L., \& Williams, G. C. 2008. Facilitating health behaviour change and its maintenance: Interventions based on self-determination theory. European Health Psychologist, 10(1), 2-5. 
SOUTO, V. 2014. A Framework for Designing Interactive Digital Learning Environments for Young People. Emerging Research and Trends in Interactivity and the HumanComputer Interface. Hershey PA.

SVINICKI, Marilla D. 2010. A guidebook on conceptual frameworks for research in engineering education. Rigorous Research in Engineering Education NSF DUE-0341127, DUE0817461.

TARIQ, Amina; TANWANI, Ajay; FAROOQ, Muddassar. 2009. User centered design of ehealth applications for remote patient management. In: 10th annual conference of the NZ ACM special interest group on human-computer interaction, CHINZ 2009, Auckland, NZ.

TOMHAVE, Benjamin L. 2005. Alphabet soup: Making sense of models, frameworks, and methodologies. George Washington University.

\section{Sobre as autoras}

Maria Luiza Bergo Demonte, Mestranda em Design, UnB, Brazil <malu.bdemonte@gmail.com> Virginia Tiradentes Souto, PhD, UnB, Brazil < v.tiradentes@gmail.com > 